\title{
Distinguish Some Textile Defects by Adopting Image Processing Methods and Intelligence Techniques
}

\author{
Iman Subhi Mohammed \\ Computer Sciences Department \\ College of Computer Science and \\ Mathematics / Mosul University/ Iraq/ \\ ImanSubhi@uomosul.edu.iq \\ DOI: 10.33899/edusj.1970.163333
}

\author{
Israa mohammed khuder \\ Computer Sciences Department \\ College of Education for Pure Sciences \\ Mosul University/ Iraq/ \\ israamoh@uomosul.edu.iq
}

Received

20/ $06 / 2018$
Accepted

$30 / 07 / 2018$

\begin{abstract}
The automatic control of the fabric is one of the important steps in the spinning and weaving industry in order to preserve the quality of the fabric. The manual methods have been used for decades to control the product using human vision. The monitoring process is very strenuous, time consuming and cost effective. To reduce the costs required there arise the needing of automated systems appearance to examine, detect and apply tissue defects.

The aim of the proposed work is to build an efficient system for detecting and classifying textile defects using advanced image processing techniques based on new methods of combining the practical implementation of image segmentation and features extraction, as well as the use of artificial intelligence techniques of neural networks for detection and classification.

The system was built in two phases: the first is the defect detection phase, and the second phase is the classification phase, where live images were collected as a textile database from the textile factory in Mosul as well as the local market. The fabrics were carefully selected and these fabrics are of different types and colors, some of these have no defect at all and some of them have up to fourteen types of defects. 560 images were collected; 280 of which were non defective fabrics, 280 were defective, and there are 20 images for every type of defect, at the defect detection phase, the statistical second-class attributes of the GLCM matrix (energy, variance, correlation, homogeneity) are extracted, while in the classification phase, the statistical first-class attributes, mean and skewness, and the geometric attribute of the total defect size. Two neural networks were used as determinants of detection and classification: the Back Propagation Neural Network (BPNN) and the Elman network. The proposed system showed a 95.7\% discrimination rate compared with other similar work in the same field.
\end{abstract}

Keyword: textile, weft direction defect, warp direction defect, Hole, Stain, statistical approaches, neural-networks, mean, Skewness, median. 


\section{تمييز بعض عيوب نسيج القماش باعتماد طرائق معالجة الصور والتقنيات الأكائية}

$$
\text { كلية التربية للعلوم الصرفة الحراء محمد خضرب }
$$

israamoh@uomosul.edu.iq DOI: $10.33899 /$ edusj.1970.163333

\author{
إيمان صبحي محمد \\ قسم علوم الحاسوب \\ كلية علوم الحاسبات والرياضيات

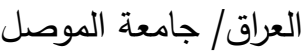 \\ ImanSubhi@uomosul.edu.iq
}

القبول

$2018 / 07 / 30$
الاستلام

$2018 / 06 / 20$

\section{الملخص}

تعد مراقبة النسيج بصورة تلقائية من الخطوات المهمة في صناعة الغزل والنسيج؛ وذلك لصيانة جودة القماش ولضمان الجودة ومطابقة المنتجات للقياسات النوعية, واستعملت الطرائق اليدوية لعقود طويلة لمراقبة المنتج وذلك باستعمال الرؤية البشرية, وتعد عملية المراقبة بهذا الأسلوب شاقة جداً ومستهلكة للوقت والكلفة، ولاختزال كل أنواع الكلفة تطلب ظهور أنظمة تلقائية لفحص عيوب النسيج وكثفها وتطبيقها. يهذف العمل المقترح إلى بناء نظام كفءٍ لكثف عيوب الأقمشة وتصنيفها؛ وذلك باستخدام تقنيات

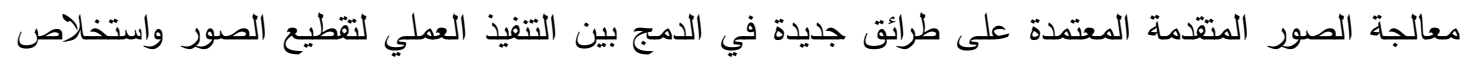

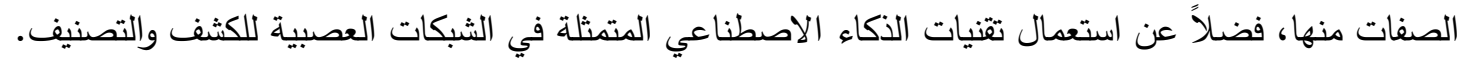
تم بناء النظام بمرحلتين: الأولى: هي مرحلة كثف العيوب، والمرحلة الثانية: هي مرحلة التصنيف،

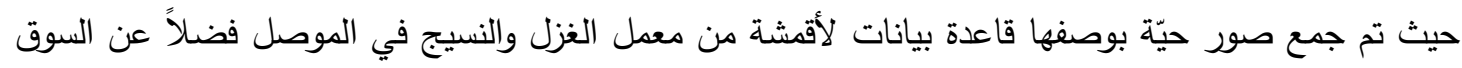

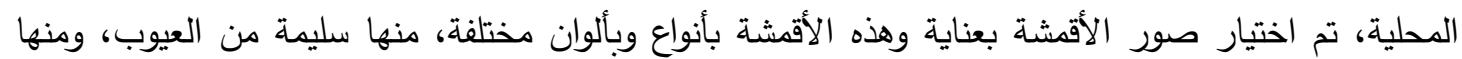

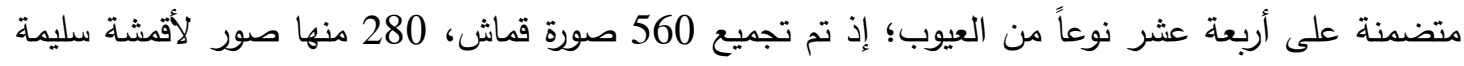
من العيب، فضلاً عن 280 صورة أخرى لأقشة معيبة، ولكل صنف من العيوب 20 صورة. في مرحلة

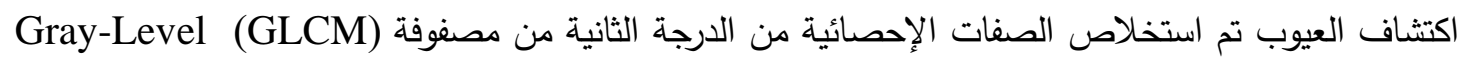
Co-Occurrence Matrix

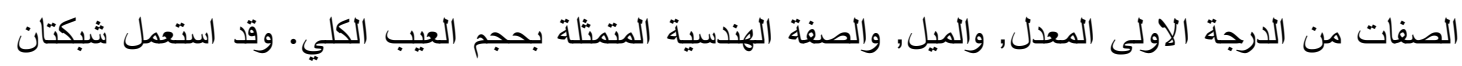

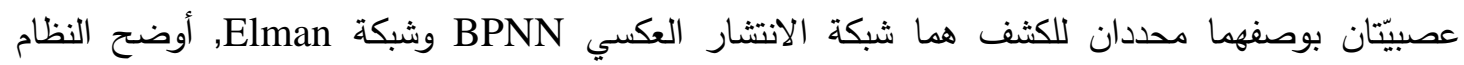
المقترح نسبة تمييز 95.7\% مقارنة بنتائج أعمال أخرى مقاربة وفي المجال نفسه.

الكلمات المفتاحية: نسيج القماش، عيب باتجاه اللحمة, وعيب باتجاه السداء, الثقب، البقعة، الطرائق الإحصائية، الثبكات العصبية، المعدل، الالتواء، الوسيط . 
أن أهية السيطرة النوعية على المنتجات الصناعية تزداد يوما بعد يوم, وتعرف السيطرة النوعية بأنها

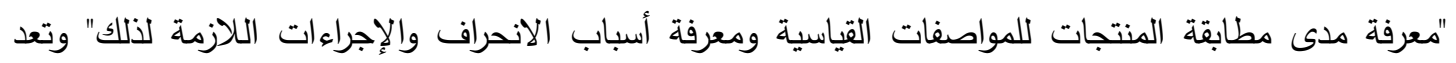
صناعة الغزل والنسيج من الصناعات التي تتطلب الجودة لتلبية رغبة المستهلك، وأمست الرقابة عليها أكثر أهمية عند المنتج للوفاء برغبة المستهلك [1]. إن مرور أية كمية غير مطابقة للمواصفات المطلوبة يقلل من سعر القماش بنسبة (45)\% - (65)\%. [2] لذا فقد أولى الباحثون اهتماما كبيرا لأنظمة فحص الغزل والنسيج

الأوتوماتيكية والتي تختصر الوقت بشكل كبير بالإضافة إلى ما تعطيه من دقة عالية في عملية الفحص [3]ة.

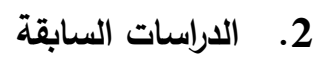

لقد تم إجراء عدد من المحاولات لجعل عملية فحص النسيج آلية وقد تركزت معظمها في الكثف عن العيب وتركز عدد قليل منها على التصنيف[1] [4] ـ. إذ استعملت عدّة طرائق للتصنيف وكانت النتائج متفاوتة الكفاءة, وفيما يأتي نبذة لبعض البحوث التي استعملت لغرض كثف وتصنيف عيوب الأقشة وبالثكل التالي:

في سنة 2007 قدم الباحثان Wheng Guang و Wang Jianxia [5 طريقة للكثف عن عيوب image distance النسيج تعتمد على تقنيات معالجة الصور وتسمى فرق البعد الحسابي للصورة difference arithmetic الذي يعكس الاختلاف بين الصورتين في اللون والإضاءة والملمس والإطار، وكان معدل دقة تحديد العيب (92)\%

في سنة 2008 تناول الباحثان K.L.Mak و P. Peng [6 عملية الكثف عن عيوب النسيج بواسطة مرشحات Gabor المثالية. وقد تم اختيار شبكة مويجات Gabor كتقنية رئيسية لاستخلاص ميزات النسيج وبالاستناد على هذه الميزات تم تصميم مرشح Gabor المثالي وبالاعتماد على قاعدة بيانات مكونة من 78 صورة متتوعة من عيوب النسيج. اقترح 2009 [7] نظاما للكثف الآلي عن عيوب الأقشة يتكون من شبكة Gabor ذلك بالاعتماد على قاعدة بيانات تتكون من 78 صورة نسيج منها 39 صورة خالية من العيوب وبقية الصور تتضمن عيوب متتوعة. وقد أثبتت نتائج الاختبار فعالية الطريقة في الكثف عن عن العيوب بنسبة $\%(97.4)$

قدم الباحث TE-LI SU وآخرون سنة 2010 [8] عملاً يوضح الكثف الأوتوماتيكي لعيوب النسيج باستخدام التحويل المويجي لاستخلاص الصفات ثم تصنيف العيوب باستعمال الثبكة العصبية

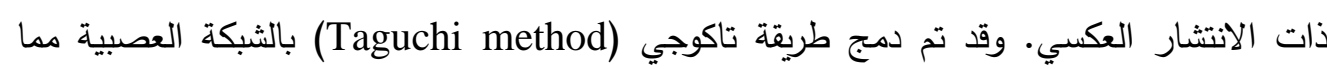

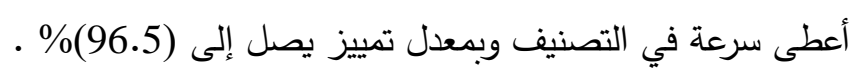

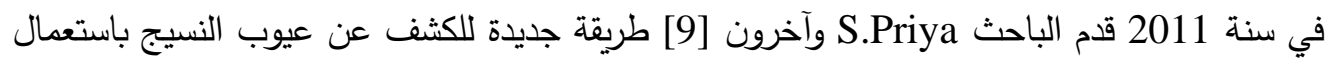
الطرائق المورفولوجية البسيطة والبعيدة عن التعقيدات الحسابية. وقد تم الاعتماد على قاعدة بيانات

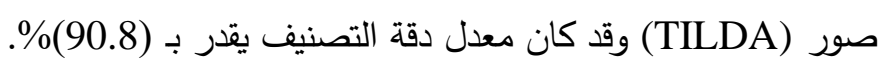


في سنة 2012 قدم الباحثان و Md. Tarek Habib M. Rokonuzzaman و بحثاً بعنوان مجموعة من الصفات الهندسية لتصنيف عيوب النسيج بواسطة الشبكة العصبية. والمتضمن حساب الصفات الهندية لمنطقة العيب مثل عرض وارتفاع نافذة العيب وعدد المناطق المعيبة والمساحة

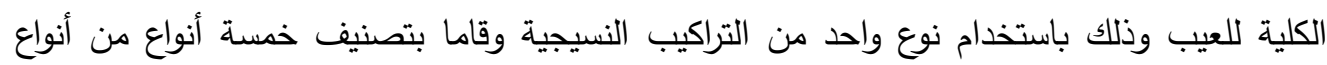

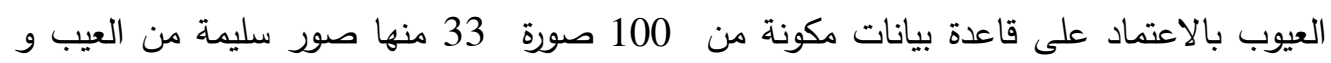
كانت دقة التصنيف (98)\% بالاعتصاد

وفي السنة نفسها قدم الباحثان Anand H. Kulkarni و Sheetal B. Patil [2] نموذج كثف دانف وتحديد عيوب النسيج الأوتوماتيكي بالاعتماد على صفات النسجة ومقاييس تواجد المستوي الرمادي Gray Level Co-occurrence Matrix (GLCM) المقترح أعطى نتائج دقيقة لتصنيف عيوب النسيج بمجموعة صغيرة من عينات التدريب وهي 10

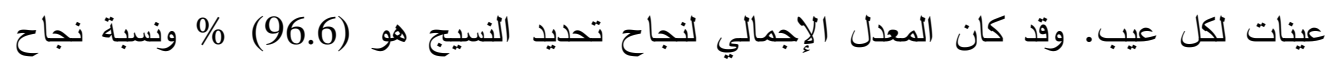

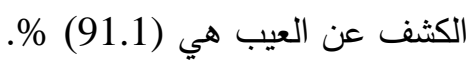
في سنة 2013 قدم Ali Javed وآخرون [10] مقارنة وتحليلا لتقنيات مختلفة في كثف عيوب

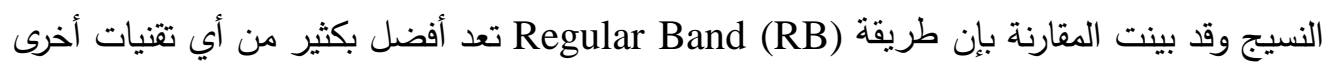
وبنسبة كثف (99.4)\% لأنها تستعمل مفهوما بسيطا من الانحراف المعياري والمتوسط المتحرك. وطريقة RB هي إحدى طرائق كشف عيوب النسيج المزخرف بكفاءة وسرعة وتستتد إلى منهج الانتظام والدورية بمعنى تكرار وحدة من الزخرفة بانتظام وأي إثارة عدم انتظام في الزخرفة تعد إثارة

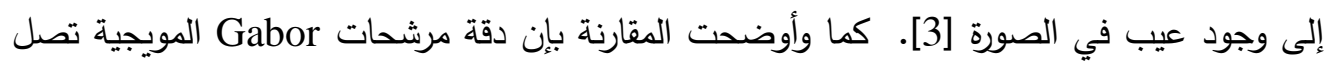
إلى (96.0)\% وبهذا تعد أفضل تقنية مستعملة لحد الآن.

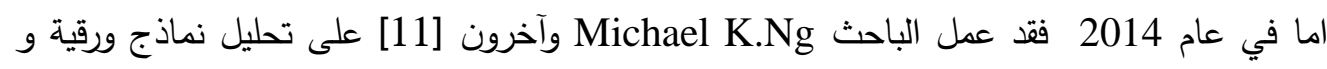
نسيجية من الاقمشة المنقوشة بعدة نقشات منها المنقط والمربعات والمنجم, وقام بفحص وتصوير العيوب في هذه الاقمة بواسطة خوارزمية التحسين المحدبة, وقد حصل على نسبة كثف تتراوح ما لكان

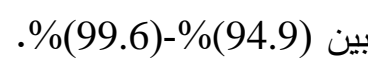

في سنة 2015 اعتمد الباحث Ali Rabhi وآخرون [12] على التجانس المحلي والثبكة العصبية

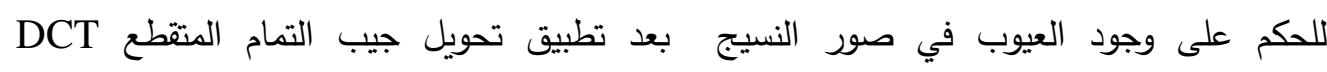

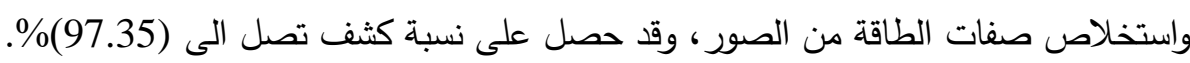

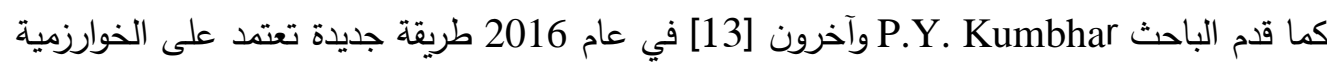
الجينية واستخلاص بعض الصفات الهندسية واستعمال آلة المتجه الداعم SVM كمصنف لعنف لعيوب النسيج وقد نجح الباحثون في تصنيف (90.0)\% من العيوب. وفي سنة 2017 اقترح الباحث Sunil Bangare وآخرون [14] طريقة لكشف عيوب النسيج باستعمال RGB وتثنيات HSV واساليب معالجة الصور وكانت دقة الكثف التي حصل عليها \%(96.15) وفي سنة 2018 تتاول الباحث Shuang Mei وآخرون [15] طريقة جديدة تعتمد على اعادة بناء الصورة بعد تصحيحها باستعمال شبكة التشفير التلقائي لتقليل الضوضاء في Gaussian pyramid, وقد اعتمد كل تصحيح كمؤشر للتنبؤ بالبكسل التالي, ومن خلال التقسيم والتجميع للمتبقي من الصورة 
المعاد بناؤها وعلى كل مستوى من مستويات الدقة حصل على النتيجة النهائية للفحص، وقد وصلت

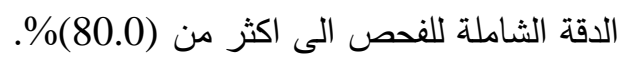

\section{3.}

يتكون القماش المنسوج Woven fabric من تشابك مجموعتين من الخيوط بشكل متعامد، المجموعة

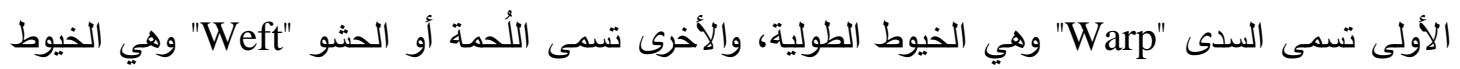

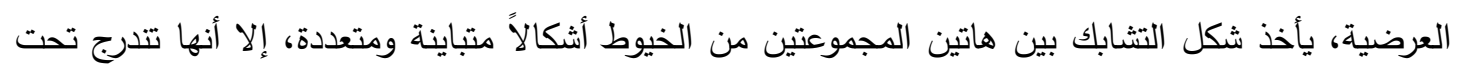

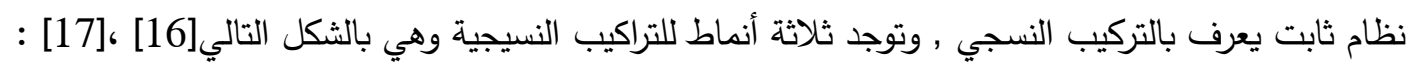
1) النستج العادي أو السادة plain weave: يعد أبسط أنواع النسج وأكثرها انتشارا. ومن الأمثلة على الأقشة

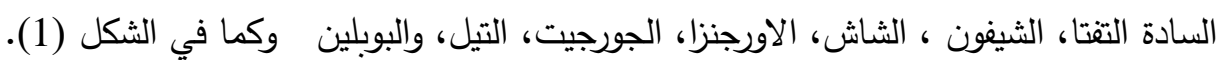

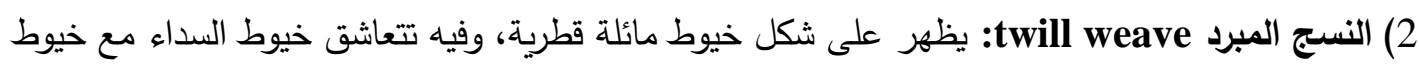

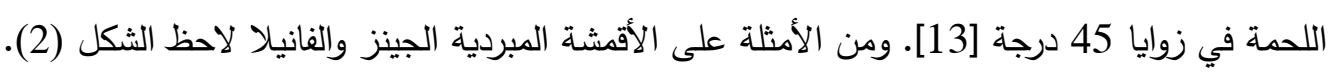
3) نستج الاطلس satin weave: يتميز بصفة عامة بسطح لامع نتيجة لتفرق مواضع تقاطع الفتل واللحمات

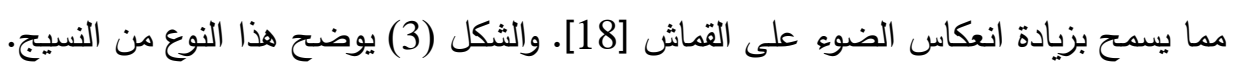

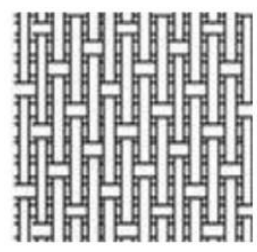

شكل (3) نسج الأطلس [17]

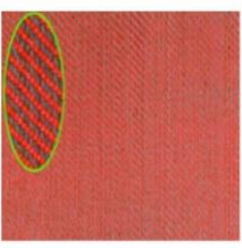

[17 [نكل (2) نسج المبرد

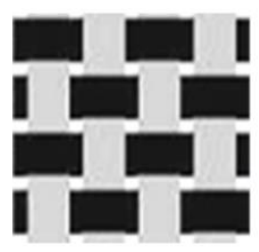

شكل (1) النسج العادي [17]

defect analysis تعليل العيوب

تعتبر عملية تحليل العيوب خطوة مهمة في عملية فحص النسيج الأوتوماتيكي، وهي تعتبر من

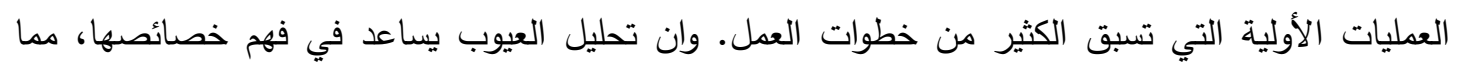

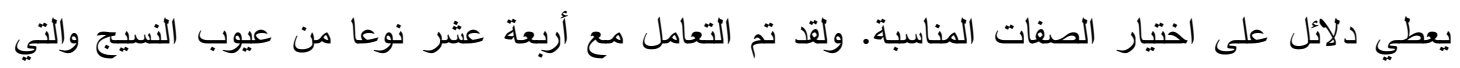

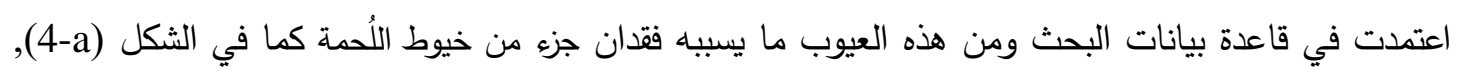

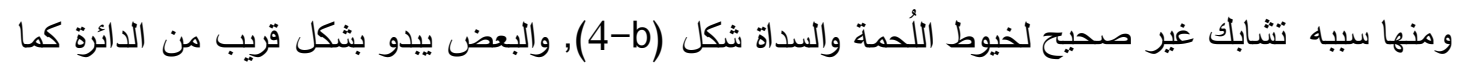

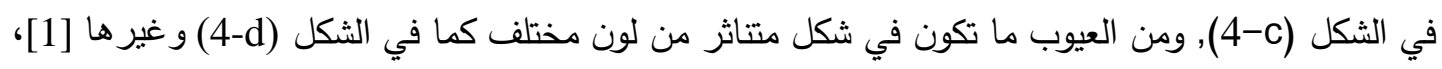
. [19]

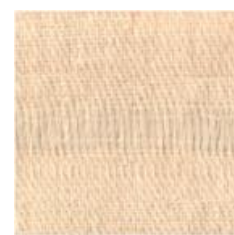

(a) (b)

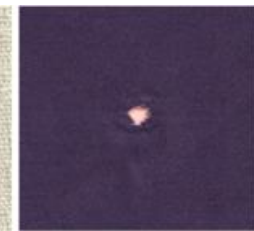

(c)

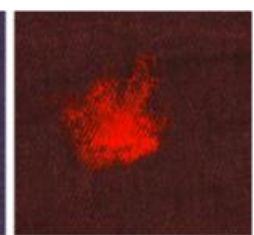

(d)

شكل (4) صور بعض انواع العيوب: (4-a) (4-b) ، Missing picks (4) Stain (4-d) ، Hole (4-c) 
5 . أساليب فحص النسيج

تصنف أساليب فحص النسيج إلى ثلاثة أصناف وهي:

* الاساليب الإحصائية Statistical approaches والتي تعتمد على السلوك الإحصائي الثابت في المناطق الخالية من العيوب. ومن أمثلتها الكثف عن الحواف edge detections والارتباط المتقاطع-cross

.[22]،[21]،correlation [20] * الاساليب الطيفية spectral approaches وتستعمل عندما تعجز الاساليب الإحصائية في كثف العيوب

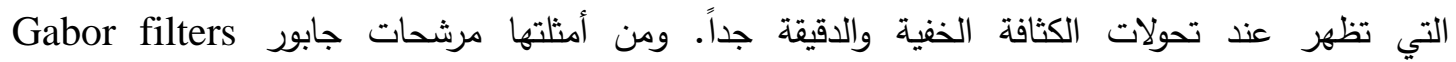
.222]،[21]،[20] * الاساليب المستندة إلى نموذج model-baesd approaches [20] وهي اساليب مناسبة لصور النسيج التي تحتوي اختلافات السطح العشوائية كذلك للأقشة المزخرفة بشكل عشوائي أو النقش اليدوي ويعد نموذج ماركوف كاوس العشوائي Gauss Markov Random Field model أحدى الاساليب في هذا المجال .[22]،[21]،[20]

6. التقنيات الأكائية إن السرعة التي يقوم الحاسب بها في عملياته الرياضية يمكن استعمالها في الكثير من المهام غير الرياضية البحتة أيضاً، فقد تكونت حقول جديدة تقع تحت مظلة الذكاء الاصطناعي كان من بينها حقل للتعرف على الأشكال والصور والنماذج، وقد دخل في تطبيقات عديدة مثل التعرف على المواقع، والتطبيقات العسكرية،

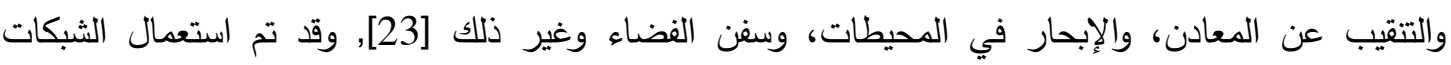
العصبية كإحدى التقنيات الذكائية لغرض الكثف عن العيوب وتصنيفها [24] ، ومن الثبكات المستخدمة في البحث ما يلي:

\section{• الثبكة العصبية ذات الانتشار العكسي BPNN) Neural Network Back Propagation):} التي تقوم على التدريب بنمط تصحيح الخطأ، حيث يتم إعادة انتشار الإشارة من الخرج إلى الدخل بشكل عكسي بوصفها إحدى مراحل التعليم ويتم خلالها ضبط أوزان الثبكة وحساب التغيرات فيها وحساب مربع الخطأ Mean (MSE) Square Error والذي ينتج من الاختلاف بين الإخراج الفعلي والإخراج المطلوب، ويستخدم الخطأ في تغيير الأوزان؛ وذلك لتقليل الخطأ تدريجياً, ويتوقف التدريب عند الحصول على أقل نسبة خطأ لكل خلية من خلايا الإخراج، فإذا اعتبر أن إشارة الإدخال e ، إذ (1) يمثل الإخراج المطلوب وإن $t_{i}$ إن هو الإخراج الفعلي، فيتم حساب دالة الخطأ كما في المعادلة $E[e]=\frac{1}{2} \sum_{i}\left(t_{i}[e]-o_{i}\right)^{2}$

• شlman وهبكة إحدى الشبكات العصبية التكرارية recurrent التي تتكون من طبقتين مع وجود تغذية الانتشار العكسي التي تتضح في إعادة إخراج الطبقة الأولى إلى إدخال الطبقة الأولى،

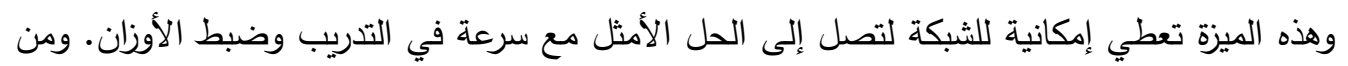


ميزات شبكة Elman أن لديها ذاكرة ديناميكية قوية؛ لذا فهي تستعمل في مجالات مختلفة كالتصنيف،

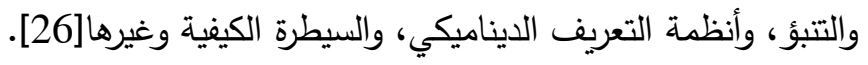

7abor 7 مرشحات

تقلد مرشحات Gabor قدرات العقول البشرية في التعرف على النسيج [27]؛ وذلك لأن دوال Gabor

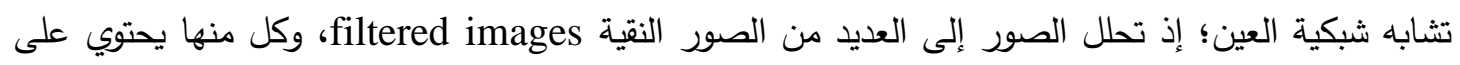
كثافات متفاوتة على نطاق ضيق من التردد والاتجاه، وتكون مرشحات Gabor ذات البعدين(D-2-2) في المجال

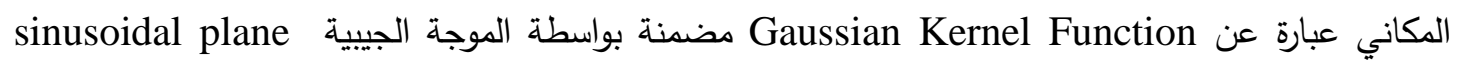
wave، ويمكن تعريف مرشحات Gabor على أنها "مجموعة من المويجات، مع كل مويجة تلتقط الطاقة بتردد

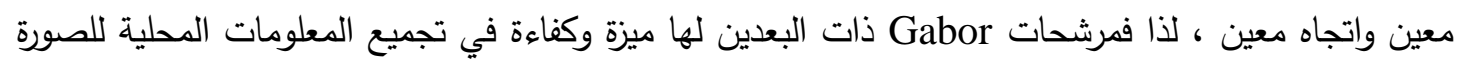

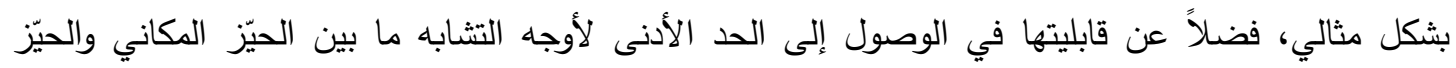
الترددي للبيانات, وقد استعملت مرشحات Gabor في العديد من تطبيقات تحليل الصور بما في ذلك تجزئة

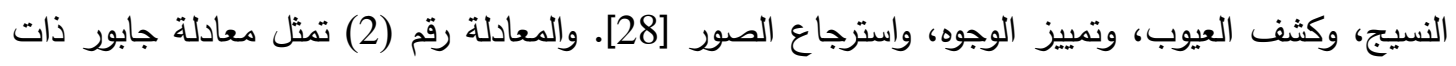
البعدين 2D-Gabor function [29] وكئن

$g w 2 D(x, y)=\frac{1}{\sigma \sqrt{\pi}} e^{-\left(\frac{\left(x-x_{0}\right)^{2}+\left(y-y_{0}\right)^{2}}{2 \sigma^{2}}\right)} e^{-j 2 \pi f 0\left(\left(x-x_{0}\right) \cos (\theta)+\left(y-y_{0}\right) \sin (\theta)\right)}$

sigmia/ standard deviation of Gaussian envelope إذ تمثل م الانحراف المعياري لمغلف كاوزين

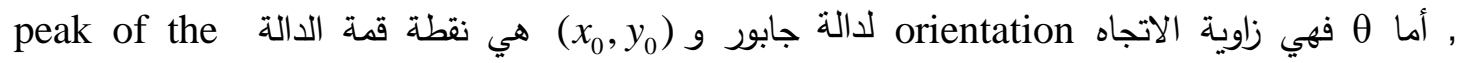

.function

8. خطوات خوارزمية العمل المقترحة

تضمنت خوارزمية العمل المقترحة مرحلتين المرحلة الأولى هي اكتشاف العيوب وتصنيفها إلى صنفين

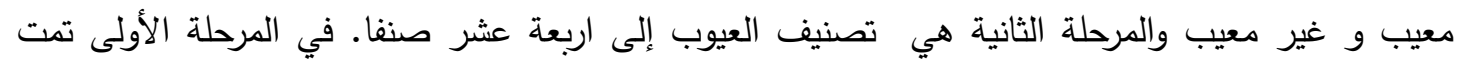

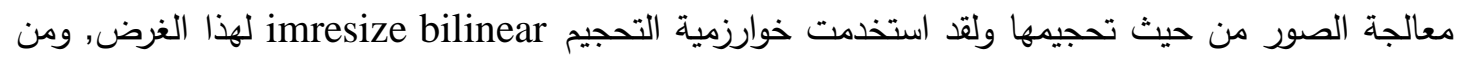
ثم معاملتها بمرشحات Gabor المتعددة؛ وذلك لإبراز العيوب الموجودة ومن ثم حساب مصفوفة GLCM واستخلاص الصفات الإحصائية من الدرجة الثانية وهي (الطاقة, التباين, درجة الارتباط و التجانس) من كل صورة لتكوين مصفوفة الادخال الى الثبكة العصبية لتقوم بتمييز صنفين من الأقمشة هما صنف أقمشة سليمة وصنف أقششة معيبة. وقد تم استخدام شبكتين عصبيتين كمصنفين هما Elman او BPNN وذلك لمقارنة نتائج الثبكتين واعتماد الثبكة الافضل من حيث زمن التدريب ودقة وسرعة الكثف والثكل (5) يوضح خطوات

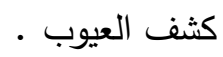



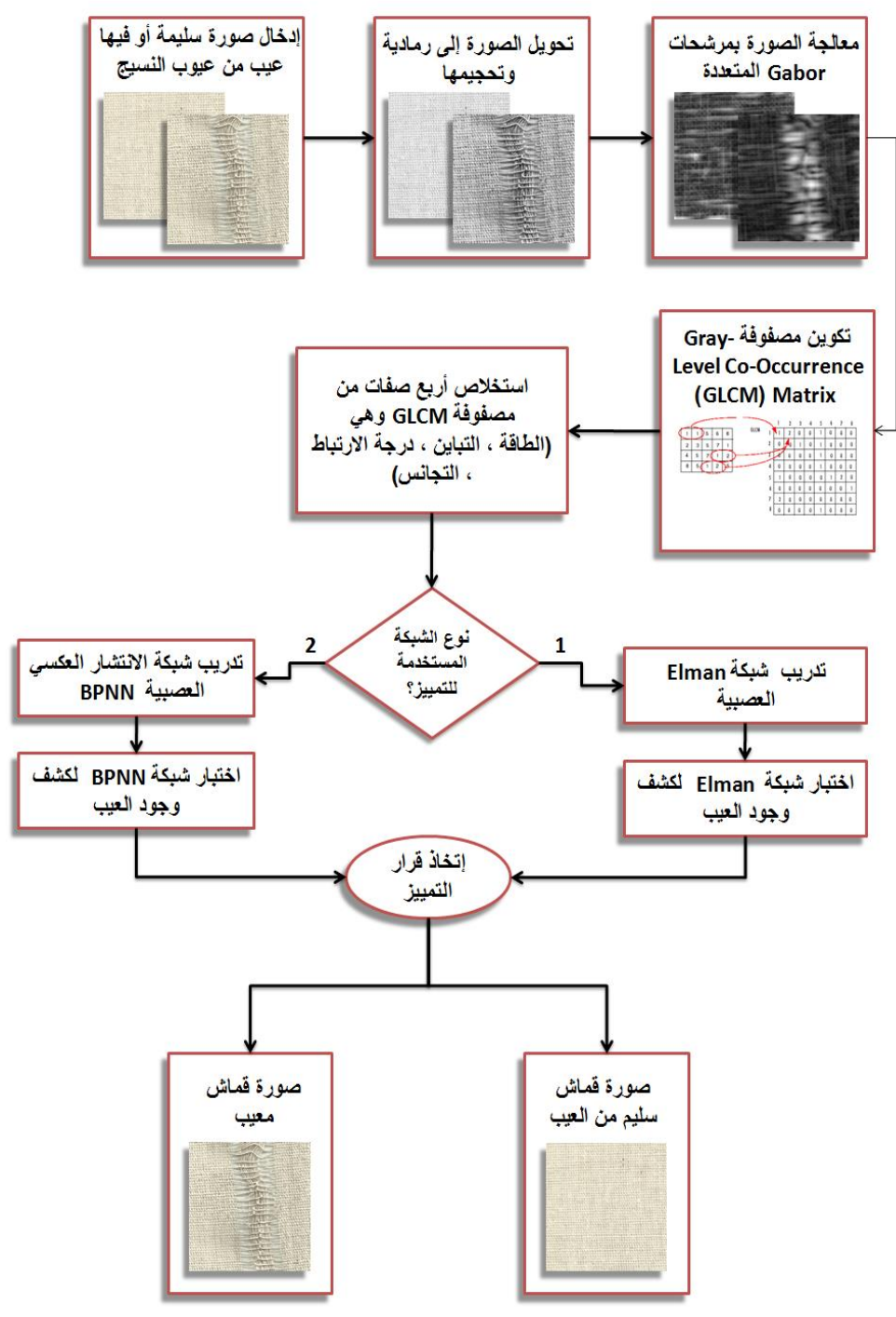

شكل (5) خوارزمية مرحلة اكتشاف العيوب

أما في المرحلة الثانية فيتم معالجة الصور المعيبة الناتجة من المرحلة الاولى، ومن ثم تقطيع هذه الصور واستخلاص الصفات الإحصائية المناسبة، لتقوم إحدى الثبكتين العصبيتين BPNN او تصنيف العيوب إلى أصنافها الاربعة عشر. أ- - تكوين قاعدة البيانات وتحجيم الصور تمت الدراسة بعد الزيارة الميدانية لمعمل الغزل والنسيج في الموصل في كانون الثاني من العام 2014

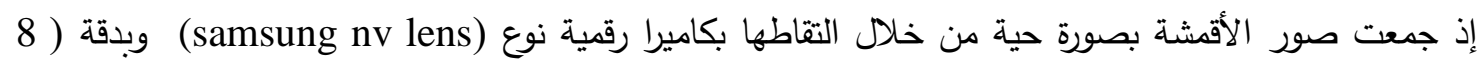
فضلا عن أخذ عينات من الأقشة من المعل ومن السوق المحلية واكتساب الصور منها عن طريق الماسح الضوئي نوع (HP) وبدقة (300 dpi) إذ تم التعرف على أنواع من الأقمشة والعيوب الموجودة فيها فضلا عن صور أخرى سليمة لتتم المقارنة بها. خزنت الصورة بملف ذي امتداد png ، ويشار من خلال [30]

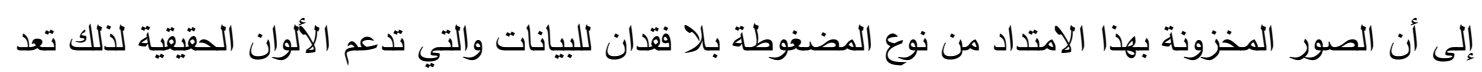


الأفضل للتعديل على الصور مقارنة بالصور ذات الامتداد jpg والتي هي من نوع المضغوطة القابلة لفقد البيانات وتقل جودتها مع كثرة الحفظ والتعديل. والثكل (6) يوضح عينات من صور قاعدة البيانات. وتمّ تجميع 560 صورة قماش؛ 280 منها صور سليمة و280 صورة أخرى فيها أنواع العيوب الأربعة عشر، ولكل صنف من العيوب 20 صورة لتتكون قاعدة بيانات حية متضمنة أنواع التراكيب النسيجية وأصناف العيوب المختلفة.
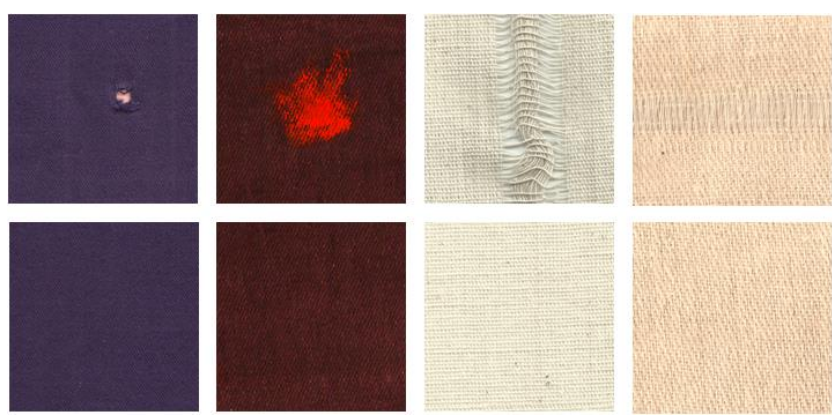

شكل (6) صور من قاعدة البيانات

ب- المرحلة الأولى - مرحلة اكتشاف العيوب في الأقمشة تتمّ في هذه المرحلة معالجة صور القماش المعيبة والسليمة وهي 560 صورة باستعمال خاصية قوة التمييز بين النسيج المعيب والنسيج السليم من العيب؛ وذلك لغرض كثف وجود العيب، وتمييز صور الأقشة بوساطة الثبكات العصبية إلى فئتين: قماش سليم وقماش معيب. وكما في الخطوات التالية:

1) المعالجة الأولية: بعد قراءة الصورة يتم تحويلها إلى صورة رمادية، لتقليل العمليات الحسابية إذ يتم تحجيم الصورة واعتماد التدرج الرمادي (gray level) في التعامل مع محتويات الصورة, ثم معاملتها بمرشحات Gabor وإبراز معالم النسجة.

2) استخلاص الصفات: حيث تستخلص صفات النسجة باعتماد طريقة حساب التبعية المكانية للمستوى الرمادي Spatial Gray Level Dependence Method (SGLDM) مقاييس تواجد المستوى الرمادي Gray-Level Co-occurrence Matrix (GLCM)، ومن ثم استخلاص الصفات من هذه المصفوفة بالكامل، وتعطي المصفوفة معلومات عن التردد النسبي لمواقع أزواج القيم الرمادية

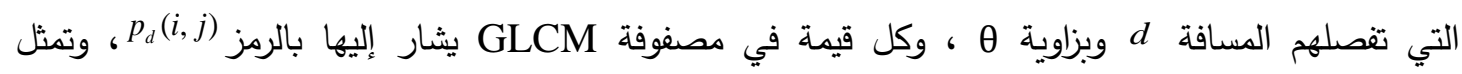

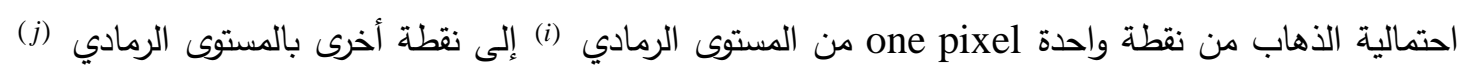
بالمسافة d والزاوية ق، وتستخلص أربع صفات مهمة في النسجة والتي تحسب من مصفوفة GLCM وهي: الطاقة، والتباين، ودرجة الارتباط، والتجانس، توصفها المعادلات من 3 الى 6 على التوالي [31]: 
- الطاقة Energy: وهي تقيس مدى اتساق النسجة بمعنى تكرار أزواج محددة من النقاط، وتحدث الطاقة العالية عندما يكون توزيع قيم المستوي الرمادي بشكل ثابت أو دوري، وتوضحها المعادلة (3): Energy $=\sum_{i} \sum_{j} p_{d}^{2}(i, j)$

- التباين Contrast: ويقيس الفرق بين أعلى القيم وأدناها لنقاط المستوي الرمادي للنسجة، وأقل تباين لصفات النسجة يكون في الترددات المكانية القليلة وتحسبها المعادلة (4):

Contrast $=\sum_{i} \sum_{j}(i-j)^{2} p(i, j)$

- الارتباط Correlation: ويقيس الاعتمادية المكانية في الصورة، وتدل قيم الارتباط العالية على خطية العلاقات بين المستويات الرمادية وأزواج نقاط الصورة، والمعادلة (5) توضح طريقة قياس صفئة الإديه الارتباط.

Correlation $=\frac{\sum_{i} \sum_{j}\left(i-\mu_{x}\right)\left(j-\mu_{y}\right) p(i, j)}{\sigma_{x} \sigma_{y}}$

$$
\begin{array}{lll}
\mu_{y}=\frac{1}{n} \sum_{i=1}^{n} j \sum_{j=1}^{n} p_{d}(i, j) & , \mu_{x}=\frac{1}{n} \sum_{i=1}^{n} i \sum_{j=1}^{n} p_{d}(i, j) \\
\sigma_{y}=\sum_{j=1}^{n}\left(j-\mu_{y}\right)^{2} \sum_{i=1}^{n} p(i, j) & , \sigma_{x}=\sum_{i=1}^{n}\left(i-\mu_{x}\right)^{2} \sum_{j=1}^{n} p(i, j)
\end{array}
$$

- زاوية العزم الثاني Angular Second Moment: وتقيس تجانس النسجة Homogeneity بإعطاء قيمة تمثل قرب توزيع عناصر مصفوفة GLCM من قطر المصفوفة، وتحسب هذه القيمة بواسطة المعادلة (6): AngularSecondMoment $=\sum_{i} \sum_{j} \frac{p(i, j)}{1+|i-j|}$

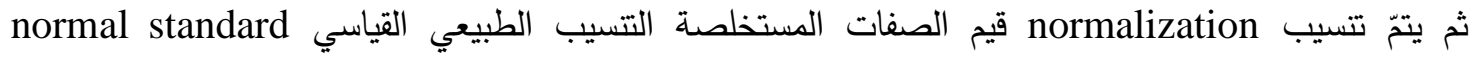
normalization (7) [31]؛ وذلك للتخلص من الفروقات الكبيرة ما بين القيم وحصر القيم بين الصفر والواحد، ثّ يتمّ وضع القيم

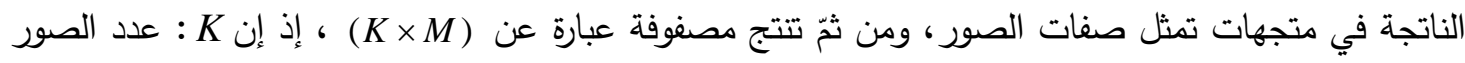
و M : عدد الصفات، ليكون لدينا مصفوفة الإدخال للشبكة العصبية.

$$
x_{i_{\text {normalized }}}=\frac{x_{i}-\mu_{i}}{\sigma_{i}}
$$


إذ تمثل $x_{i} x_{i_{\text {normalized }}}$ التسيب.

3) كثف العيب : يتم في هذه المرحلة التعامل مع صور الفحص لغرض تحديد وكثف وجود العيب من عدم

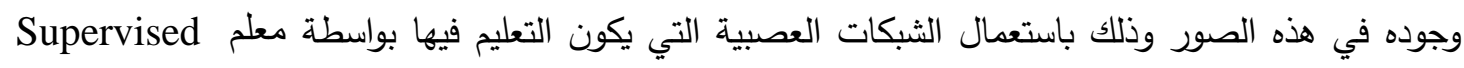

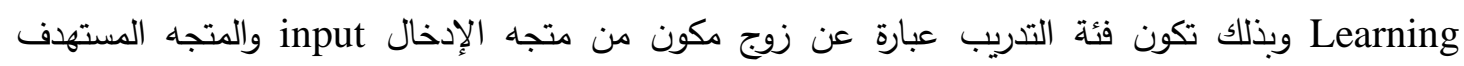

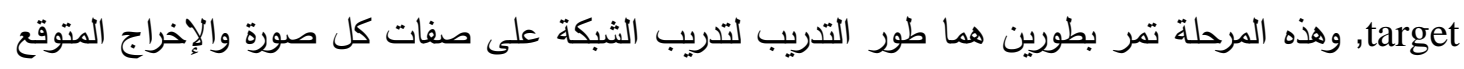
لها، وقد خصصت في هذه المرحلة 420 صورة كعينـة للتدريب, وطور الاختبار ليتم من خلاله اختبار الشبكة على صور جديدة لم تتعلم عليها الثبكة تسمى صور الاختبار وقد تم تهيئة 140 صورة كعينة للاختبار. في

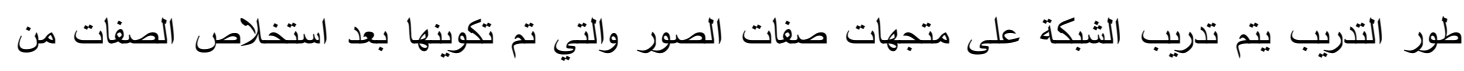
الصور • وذلك لغرض تعليم الشبكة لتتمكن من الكشف عن وجود العيب وتصنيف كل صورة إما إلى صورة

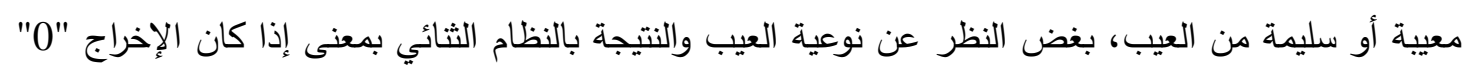
فهي صورة خالية من العيب إما إذا كان الإخراج "1" فالصورة معيبة. في طور الاختبار يتم اختبار الثبكة العصبية لكشف العيب وتتبع نفس خطوات المعالجة الأولية واستخلاص الصفات لغاية الحصول على متجه

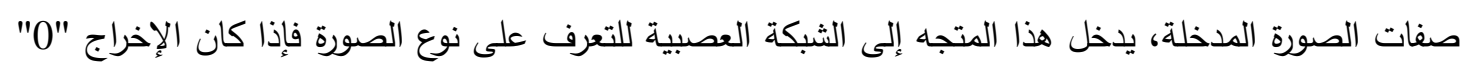

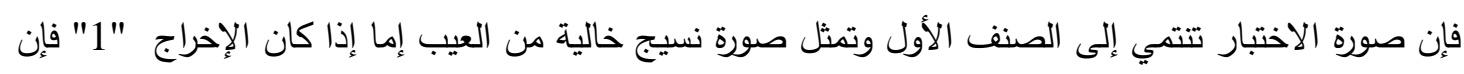

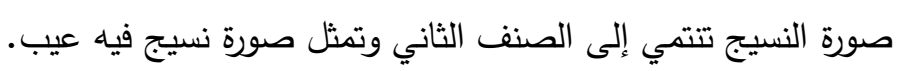

ج- المرحلة الثانية- تصنيف عيوب النسيج ويتم في هذه المرحلة معالجة صور النسيج المعيبة فقط وهي 280 صورة وتصنيفها بواسطة الثبكات العصبية BPNN و Elman إلى أربع عشرة فئة تمثل أصناف العيوب. وكما في الخطوات الآتية: 1) المعالجة الأولية و قطع منطقة العيب (ROI- Region Of Interest) من الصور: بعد قراءة صورة النسيج المعيبة وتحجيمها يتم معاملتها بمرشحات Gabor، وذلك لإبراز وتحديد العيوب الموجودة في الصورة. بعدها يتم تحويل الصورة الرمادية الناتجة من Gabor إلى صورة ثنائية، وذلك بتحويل كل بكسل في الصورة الرمادية والمشار له [0,1 يشير إلى "اسود" ويشير"1" إلى "ابيض" وتدعى هذه العملية بعملية Binarization. وهناك عدة أساليب لتتفيذ عملية Ginarization منها Global fixed threshold وهي المستعملة هنا, إذ يتم استعمال حد عتبة ثابتة $b_{i}=1 \quad$ if $\quad x_{i} \geq 0.5 \quad \& \quad b_{i}=0 \quad$ if $\quad x_{i}<0.5$ كالأتي [32]:

وبتطبيق مرشح المعدل Averageing (7*7) يتم إزالة الثوائب من الصورة الثنائية الناتجة وتحسين

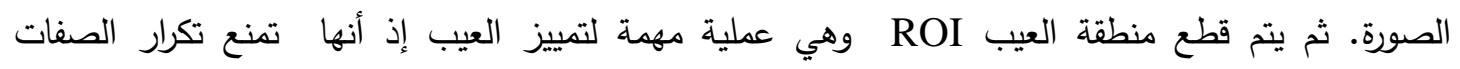
المستخلصة وهذا التكرار يحصل إذا تم أخذ صفات الصورة بالكامل بالإضافة إلى أنها تقلل من أبعاد مصفوفة

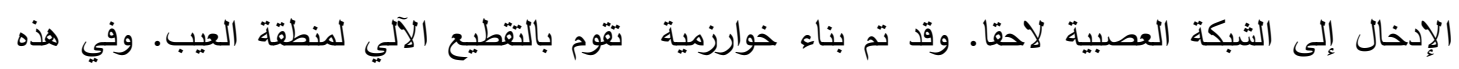

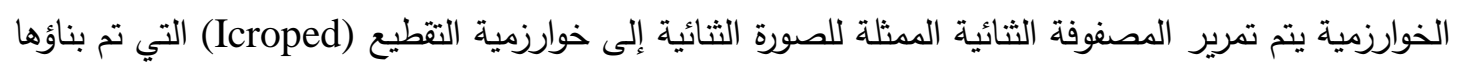


لتقوم بالتقطيع الآلي على خطوتين متتاليتين في الخطوة الأولى: يتم التقطيع باتجاه الأعمدة لقطع الأسطر غير

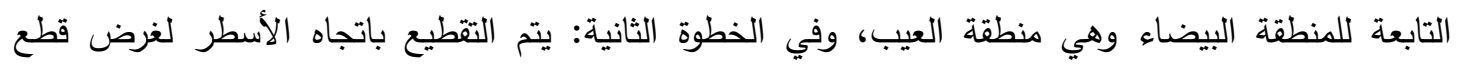
الأعمدة المحيطة بمنطقة العيب، وبهذا يكتمل استقطاع الجزء المهم من صورة النسيج وهو جزء العيب.

2) استخلاص الصفات و تصنيف العيوب: يتم حساب الحجم الكلي لمنطقة العيب واعتبارها كإحدى الصفات الهندسية للصورة. ثم يحدد محيط منطقة العيب بعامل كاني لكثف الحواف canny operators. يليه تقطيع

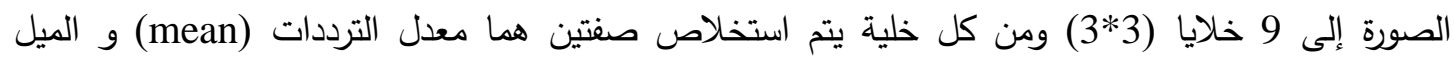
ليكون لدينا (Skewness) الحجم الكلي لمنطقة العيب فيكون مجموع الصفات المستخلصة من الصورة 19 صفة. يتم ترتيب صفات صنيات صور العيوب في مصفوفة ثنائية عدد صفوفها يمثل عدد الصور وعدد أعمدتها يمثل عدد الصفات وهكذا تتكون مصفوفة الإدخال إلى الثبكة العصبية لغرض تصنيف أنواع العيوب. ويككن حساب العزم الثالث المتمثل بالميل skewness $=\frac{1}{N}\left(\frac{\sum_{i-1}^{N}\left(X_{i}-\bar{X}\right)^{3}}{\sigma^{3}}\right)$ (Skewness)

ثم تصنف العيوب إلى أصنافها الاربعة عشر وذلك باستخدام احدى الشبكات العصبية, شبكة الانتشار

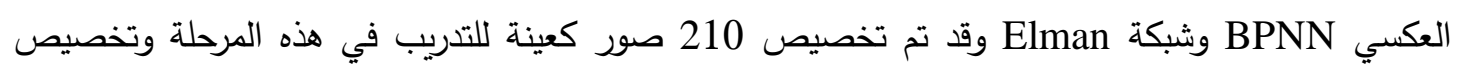

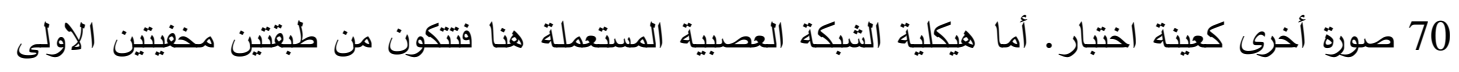

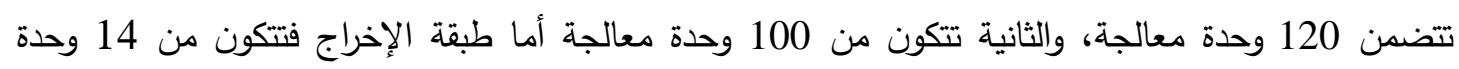

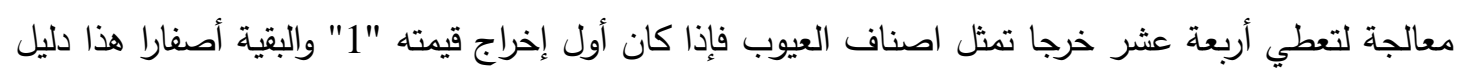

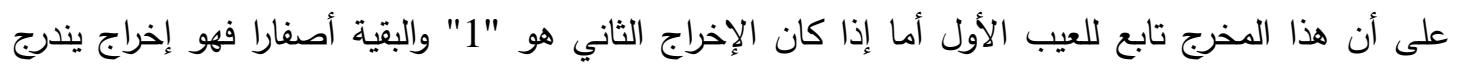
تحت العيب الثاني وهكذا بقية العيوب.

الطور الأول- تدريب الثبكة العصبية: يتم تدريب الثبكة على متجهات صفات الصور المعيبة والتي تم

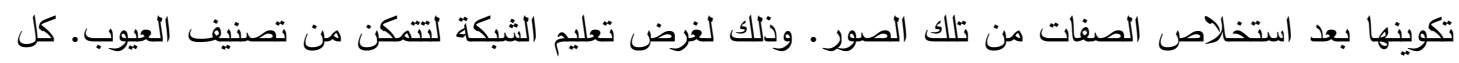

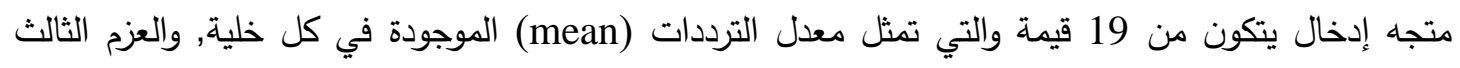
المتمثل بالميل (Skewness) لكل خلية, بالإضافة إلى صفة مجم منطقة العيب وبعد تتسيب هذه الصفات بالنسبة لأعلى قيمة وبواقع 9 مناطق لكل صورة. (normalization)

الطور الثاني- اختبار الثبكة العصبية لتصنيف الصور: وتتبع نفس خطوات المعالجة الأولية واستخلاص الصفات لغاية الحصول على متجه صفات الصورة المدخلة, ثم يتم إدخال هذا المتجه إلى الثبكة العصبية للتعرف على نوع الصورة وشكل العيب وارجاعه الى صنفه الذي ينتمي اليه. الثكل(7) يوضح كيف تتم عملية تدريب الثبكة العصبية وخزن معاملاتها والاوزان المثالية في طور التدريب ومن ثم استرجاعها في طور الاختبار لفحص الصور غير المدربة على الشبكة مسبقا. 


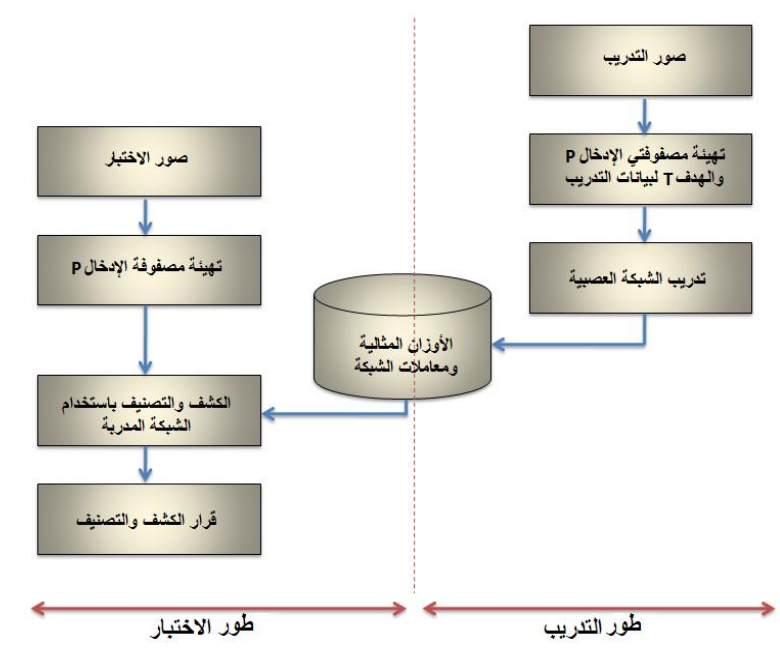

شكل (7) المخطط الصندوقي لطوّرَي التدريب والاختبار في الثبكة العصبية

\section{9. النتائج ومناقشتها}

أ- من خلال التطبيق العملي للخوارزمية المقترحة تم تدريب شبكة Elman العصبية لاكتشاف العيب بعد

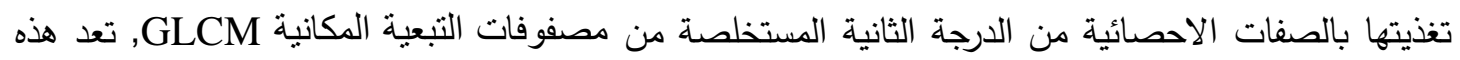
الطريقة في استخلاص الصفات مميزة من حيث قلة عدد الصفات المستخلصة بالإضافة إلى جودتها والتي قللت العبء الحسابي المتكرر عن الثبكة العصبية ما ادى الى سرعة في عملية التمييز .

بـ تم تدريب الثبكات العصبية على 420 صورة منها 210 صورة نسيج طبيعي سليم من العيوب 210 صورة لنسيج يحوي على عيوب النسيج المختلفة وتم تهيئة 140 صورة غير مدربة على الثبكة العصبية منها 70 صورة من صور النسيج السليمة من العيوب و 70 أخرى فيها عيوب مختلفة وبعد اختبار الثبكات العصبية

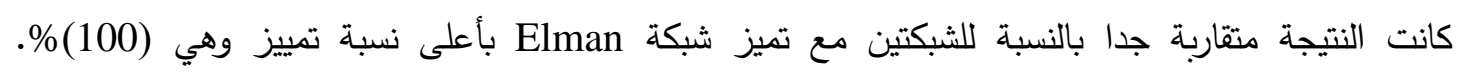
والجدول (1) يوضح نتائج الشبكتين BPNN و

جدول (1) نتائج فحص صور الاختبار على شبكة BPNN و Elman

\begin{tabular}{|c|c|c|c|c|}
\hline الوقت بالثو اني لاختبار 140 & النسبة الكلية & نسبة اختبار 70 صورة & نسبة اختبار 70 صورة & نوع الثبكة \\
\hline 105.5570 & $100 \%$ & $100 \%$ & $100 \%$ & Elman \\
\hline 113.4430 & $99.2857 \%$ & $100 \%$ & $98.5714 \%$ & BPNN \\
\hline
\end{tabular}

الشكل رقم (8) يوضح نتائج اختبار الصور غير المدربة على شبكة BPNN و Elman الاعتماد على جدول 


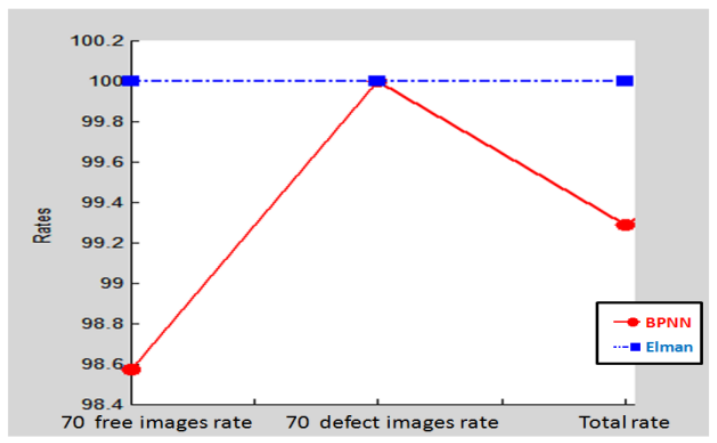

شكل (8) نتائج عملية كثف وجود العيب في صور الاختبار على شبكتي BPNN و Elman

والثكل (9) يوضح نسبة التمييز الكلية لكل من الثبكتين فضلا عن مقدار الوقت المستتفذ لاختبار 140 صورة من الصور غير المدرية والمعدة لغرض فحص كفاءة الثبكتين في التمييز , بالاعتماد على جدول (1).

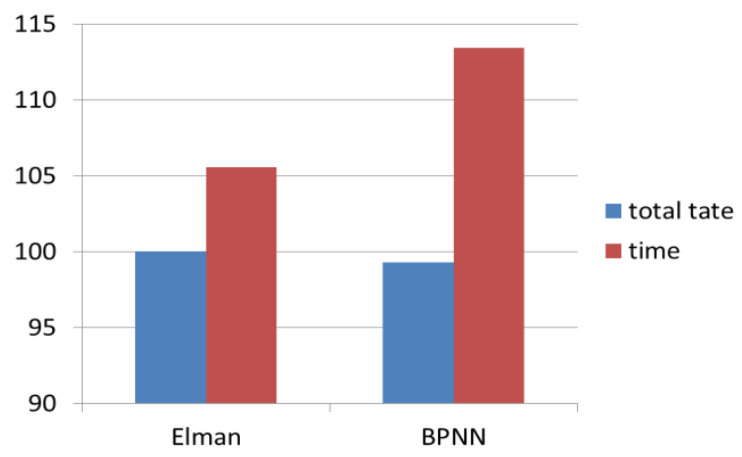

شكل (9) نتائج عملية كثف وجود العيب في صور الاختبار على شبكتي BPNN و Elman

ج - تم اختبار 70 صورة من الصور المعيبة بواقع 5 صور من كل نوع من العيوب الأربعة عشر على

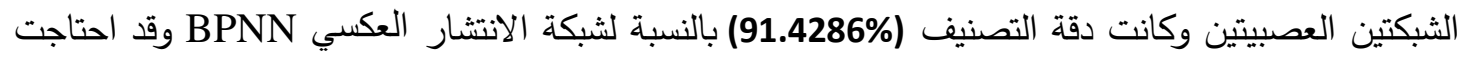

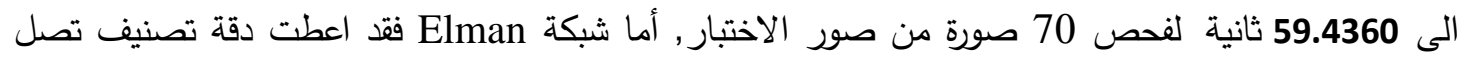
الى (87.1429\%) وكان الوقت اللازم لفحص 70 صورة يساوي 59.0150 ثانية. والثكل (10) يعطي مخططا توضيحيا عن النسبة الكلية للتصنيف في كلا الشبكتين بالإضافة الى الوقت بالثواني اللازم لتصنيف 70 صورة من صور الاختبار .

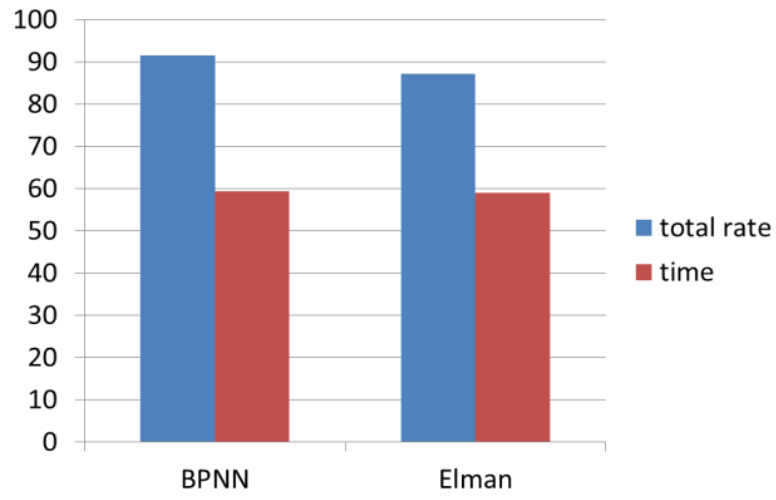

شكل (10) نتائج عملية تصنيف العيوب في صور الاختبار على شبكتي BPNN و Elman 


$$
\text { والجدول(2) يوضح نتائج الثبكات العصبية BPNN و Elman في مرحلة تصنيف العيوب. }
$$

جدول (2) نتائج عملية تصنيف العيوب لبيانات التدريب والاختبار على الثبكتين

\begin{tabular}{|c|c|c|c|c|c|c|}
\hline \multicolumn{4}{|c|}{ صور الاختبار } & \multicolumn{2}{|c|}{ صور التندريب } & \multirow{3}{*}{ 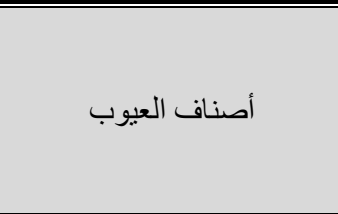 } \\
\hline \multicolumn{2}{|c|}{ اختبار 70 صورة غيرة مدربة BNNN } & \multicolumn{2}{|c|}{ اختبار شبكة 70 صورة غير مدربة } & \multirow{2}{*}{ دقة 210 صورة التصنيف } & \multirow{2}{*}{ دقة 210 صورزة عدبة على } & \\
\hline 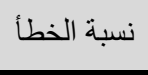 & دقة التصنيف & 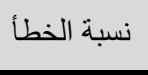 & دقة التصنيف & & & \\
\hline $0 \%$ & $100 \%$ & $20 \%$ & $80 \%$ & $100 \%$ & $100 \%$ & العيب1 (Broken ends) \\
\hline $0 \%$ & $100 \%$ & $0 \%$ & $100 \%$ & $100 \%$ & $100 \%$ & العيب2 (Broken pattern) \\
\hline $0 \%$ & $100 \%$ & $0 \%$ & $100 \%$ & $100 \%$ & $100 \%$ & العيب3 (coloured flecks) \\
\hline $20 \%$ & $80 \%$ & $20 \%$ & $80 \%$ & $100 \%$ & $100 \%$ & العيب4 (Cut \& tear) \\
\hline $0 \%$ & $100 \%$ & $0 \%$ & $100 \%$ & $100 \%$ & $100 \%$ & العيب5 (Double ends) \\
\hline $0 \%$ & $100 \%$ & $0 \%$ & $100 \%$ & $100 \%$ & $100 \%$ & العيب6 (Float) \\
\hline $0 \%$ & $100 \%$ & $20 \%$ & $80 \%$ & $100 \%$ & $100 \%$ & العيب7 (Hole) \\
\hline $20 \%$ & $80 \%$ & $0 \%$ & $100 \%$ & $100 \%$ & $100 \%$ & العيب8 (Knots) \\
\hline $40 \%$ & $60 \%$ & $40 \%$ & $60 \%$ & $100 \%$ & $80 \%$ & العيب9 (Missing picks) \\
\hline $0 \%$ & $100 \%$ & $20 \%$ & $80 \%$ & $100 \%$ & $100 \%$ & العيب10 (Reed Marks) \\
\hline $0 \%$ & $100 \%$ & $100 \%$ & $0 \%$ & $100 \%$ & $13.3333 \%$ & العيب11 (Slub) \\
\hline $20 \%$ & $80 \%$ & $0 \%$ & $100 \%$ & $93.333 \%$ & $100 \%$ & العيب12 (Stain) \\
\hline $0 \%$ & $100 \%$ & $0 \%$ & $100 \%$ & $100 \%$ & $100 \%$ & $\begin{array}{c}\text { (Untrimmed 134 العيب) } \\
\text { Loose threads) } \\
\end{array}$ \\
\hline $0 \%$ & $100 \%$ & $0 \%$ & $100 \%$ & $100 \%$ & $100 \%$ & العيب14 (Weft Bar) \\
\hline $8.5714 \%$ & $91.4286 \%$ & 12.8571 & $87.1429 \%$ & $99.5238 \%$ & $93.8095 \%$ & النسبة الكلية \\
\hline
\end{tabular}

والثكل رقم (11) يوضح نتائج تصنيف عيوب نسيج القماش على شبكة BPNN بالاعتماد على جدول رقم

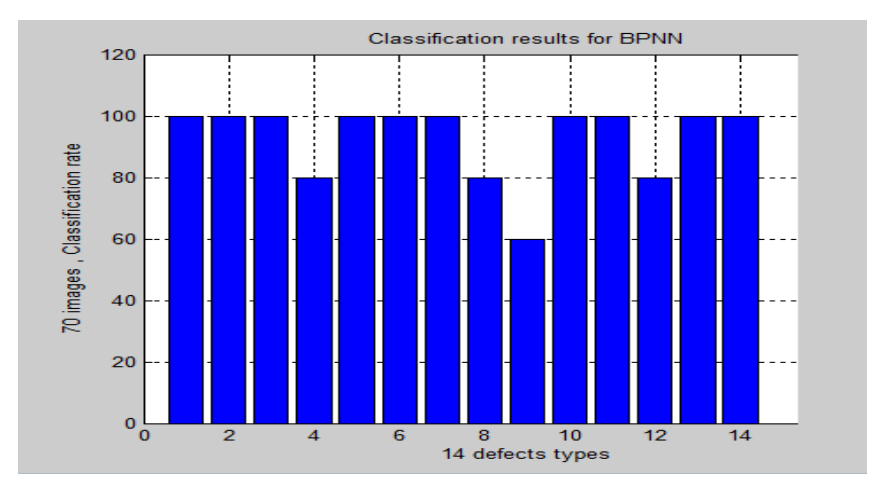

شكل (11) نتائج تصنيف عيوب نسيج القماش بشبكة BPNN

والشكل رقم (12) يوضح نتائج تصنيف عيوب نسيج القماش على شبكة Elman بالاعتماد على جدول رقم 


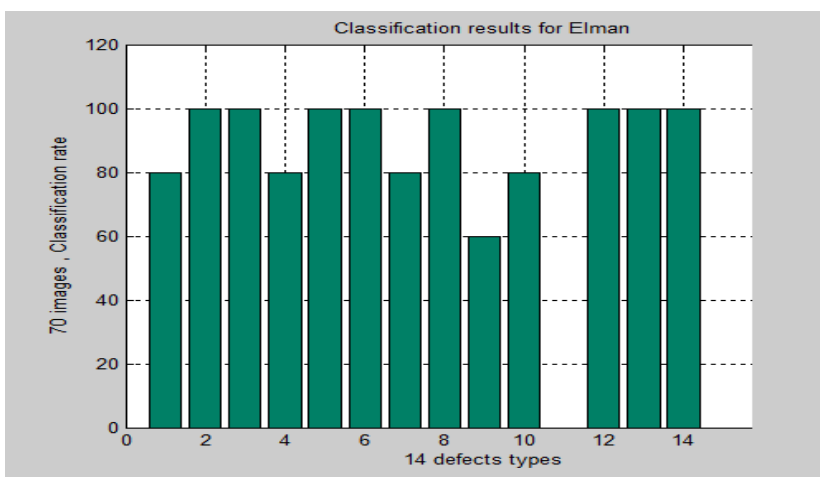

شكل (12) نتائج تصنيف عيوب نسيج القماش بشبكة Elman

والثكل (13) يمثل نسب التصنيف الكلية لبيانات التدريب وبيانات الاختبار ومعدل الخطأ لكل من الشبكتين العصبيتين BPNN و Elman

بابكة Elman

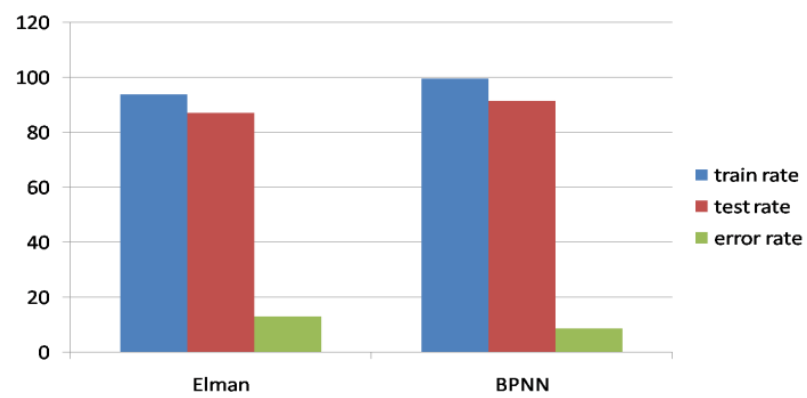

شكل (13) نتائج تصنيف عيوب نسيج القماش

10 10 الاستنتاجات

بعد المطالعة النظرية للعمل المقترح والتطبيق العملي الذي تم فيه دمج طرائق معالجة الصور من

خلال استعمال مرشحات Gabor واستخلاص الصفات من مصفوفة التبعية الدكانية GLCM مع التقنيات الذكائية باستعمال الثبكة العصبية المتمثلة بشبكة Elman للكثف عن العيوب والثبكة العصبية ذات الانتشار العكسي BPNN لتصنيف العيوب في صور نسيج القماش. تم الحصول على نسبة تمييز تصل إلى 95.7 بعنه وهذه النسبة تعتبر ممتازة مقارنة بنسب أخرى في بحوث المجال نفسه, إلا أن العمل لا يمكن أن يصل الكمال لأن لكل أسلوب محاسنه ومساوئه والمتمثلة هنا بصعوبة الحصول على صور الأقشة والعيوب من قواعد بيانات

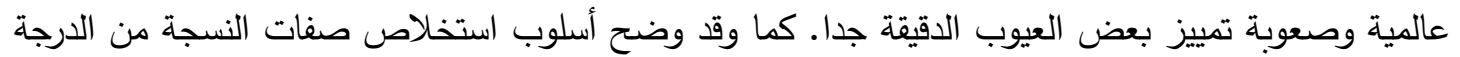
Gray-Level Co-occurrence (GLCM) الثانية بعد حساب مصفوفة مقاييس تواجد المستوى الرمادي Spatial Gray Level (SGLDM) المتمثل بخوارزمية حساب التبعية المكانية للمستوى الرمادي Matrix Dependence Method العمل. وقد تم اعتماد صور حية من الأقشة السليمة من العيوب والأقمة المعيبة لتتاسب العمل المقترح والتي

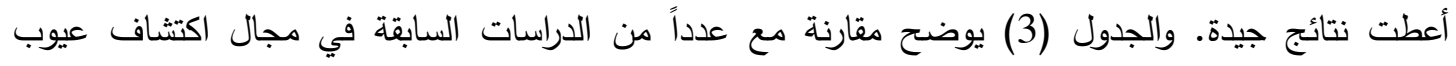
الأقشة وتصنيفها والطرائق المستخدمة مع نسبة التمييز . 
جدول رقم (3) مقارنة مع الدراسات السابقة

\begin{tabular}{|c|c|c|c|c|c|}
\hline نسبة التمييز & قاعدة البيانات & السنة & الطريقة المستخدمة & أسماء الباحثين & ت \\
\hline $96.7 \%$ & 39 صورة قماش خالية من العيوب 78 صورة قماش فيها 32 نوعاً من & 2008 & $\begin{array}{c}\text { مرشحات Gabor المثالية } 1 \text { مبكة مويجات Gabor } \\
\text { (GWE) }\end{array}$ & $\begin{array}{l}\text { \& K.L.Mak } \\
\text { P. Peng }\end{array}$ & 1 \\
\hline $96.5 \%$ & 200 صورة قماش فيها 4 أنواع من 50 صورة من كل نوع & 2010 & الانتشار العكسي المويجي + شبكة & TE-LI SU وآخرون TE & 2 \\
\hline $98 \%$ & خالية من العيوب والبقية فيها 5 أنواع 33 صورة & 2012 & شبكة الانتشار العكسي & $\begin{array}{l}\text { \& Md. Tarek } \\
\text { M. Habib } \\
\text { Rokonuzzaman }\end{array}$ & 3 \\
\hline $91.1 \%$ & قماش خالية من العيوب و 150 صورة قماش منها 30 صورة صورة 120 صورة & 2012 & $\begin{array}{c}\text { الاحتمالية GLCM } \\
\text { PNN الشبكة العصبية }\end{array}$ & $\begin{array}{c}\text { Anand H. } \\
\text { \& Kulkarni } \\
\text { Sheetal B. Patil }\end{array}$ & 4 \\
\hline $96 \%$ & 39 صورة قماش خالية من العيوب 78 صورة قماش قماش فيها 32 نوعاً من & 2013 & Regular Band & Ali Javed وآخرون & 5 \\
\hline $90.0 \%$ & --------- & 2016 & الخوارزمية الجينية وآلة المتجه & $\begin{array}{l}\text { P.Y. Kumbhar } \\
\text { وآخرون }\end{array}$ & 5 \\
\hline $96.15 \%$ & 26 صورة قماش خالية من العيوب 26 & 2017 & 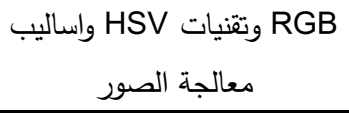 & $\begin{array}{c}\text { Sunil Bangare } \\
\text { وآخرون }\end{array}$ & 7 \\
\hline $80.0 \%$ & TELDA database & 2018 & $\begin{array}{l}\text { شبكة التشفير التلقائي + } \\
\text { Gaussian pyramid } \\
\end{array}$ & Shuang Mei وآخرون & 8 \\
\hline $95.7 \%$ & من العيوب والباقي صورة قماش منها 280 صأقمشة فيهة 14 نوعاً من العيوبة & 2018 & 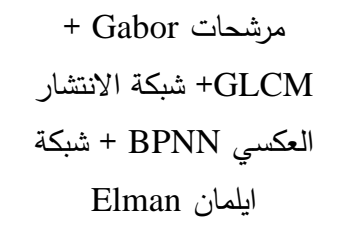 & إيمان صبحي محمد \& إسراء محمد خضر & ) \\
\hline
\end{tabular}

\section{المصادر}

[1] Tarek Habib MD. and Rokonuzzaman M., ISRN Artificial Intelligence, 2012:1-16 (2012).

[2] Anand H. K. and Sheetal B. P., (IJLTET), (2012).

[3] Rao Ananthavaram R. K. , Srinivasa O. Rao and Krishna Prasad MHM, (IJCA) ,(2012).

[4] Dewan R. and Aggarwal M. , (AMIERJ), (2012).

[5] Guang Z. and Jianxil W. , China (ICEMI),(2007).

[6] Mak K.L. and Peng P., (WASET ),(2008).

[7] Mak K.L. ,Peng P. and Yiu K.F.C.,Image Vis Comput. , 27:1585-1592, (2009).

[8] Te-Li Su, Hua-Wei Chen, Gui-Bing Hongand Chih-Ming Ma, International Conference on Wavelet Analysis and Pattern Recognition ,(2010). 
[9] Priya S., Ashok kumar T. and Varghese Paul Dean, International Conference on Signal Processing, Communication Computing and Networking Technologies (ICSCCN), (2011).

[10] Javed A., Ahsan Ullah M., and Ur-Rehman A. , (IJIGSP), 40-45 (2013).

[11] Michael K. Ng, Henry Y. T. Ngan, Senior Member, Xiaoming Yuan, and Wenxing Zhang,IEEE Trans on Autom Sci and Eng, (2014).

[12] Rebhi A., Benmhammed I., Abid S. and Fnaiech F., IEEE Photonics J, (2015).

[13] Kumbhar P. Y. , Mathpati T. , Kamaraddi R. and Kshirsagar N. , (IJREST), 3:3 (2016).

[14] Bangare S. L., Dhawas N. B., Taware V. S., Dighe S. K., Bagmare P. S., (IJRAT), 5:6 (2017).

[15] Shuang Mei , Yudan Wang, and Guojun Wen , 18:1064 (2018).

[16] Salah Hassan Naji., Journal of University of Babylon, 20:1:174-182 (2012). (In Arabic)

[17] Peng Pai R., Ph.D. Dissertation, The HKU Scholars, University of Hong Kong, (2006).

[18] Zalat A. El-S. , El-Gamal M. A. , Ibrahim N. H., (QERJ) ,18 (2010). (In Arabic)

[19] Maroš Tunák, Aleš Linkaand Petr Volf ,Quality \& Productivity Research Conference,(2009).

[20] Kumar A. and Khas H. , IEEE Transactions on Industrial Electronics, 55:1:348363, (2008).

[21] Mahajan P.M. , Mahajan J.T. and Patil P.M, Advances in Computational Research, 1:2:18-29, (2009).

[22] Priyanka M. Shanbhag, Manish P. Deshmukh and Shekhar R. ,(GJEDT), 1:2:s246, (2012).

[23] HOGGARS. G., "Mathematics of Digital Images Creation, Compression, Restoration, Recognition", Cambridge University Press, New York, (2006).

[24] Mohammed I.S. , AIP Conference Proceedings 2096,020005- 020005-7 (2019).

[25] Tom Mitchell, "Machine Learning", McGraw Hill, (1997).

[26] Zhiqiang Zhang, Shangce Gao, Gang Yang, Fangjia Li and Zheng Tang, INT J INNOV COMPUT I, 5:10:2997-3011, (2009).

[27] Arivazhagan S., Ganesan L., and Bama S., MACH VISION APPL 16:6:356363, (2006).

[28] Zhang D. , Wong A. , Indrawan M., Lu G., First IEEE Pacific-Rim Conference on Multimeadia, Australia: 392-395, (2000).

[29] Mohammed I.S.and khuder I. M. , (KUJSS), 3:529-555(2017) (In Arabic).

[30] Mohammed I.S. and Alhamdani I. M. , (IJECE), v9:i5: 4277-4286(2019).

[31] Hassan M. Elragal, Proceeding of 23rdNational Radio Science Conference (NRSC): 14-16, (2006).

[32] Maya R. Gupta , Nathaniel P. Jacobsonand Eric K. Garcia, ,INT JPATTERN RECOGN 40:389 - 397, (2007).

[33] Badran A., Jassem M. A. , Mahmoud M. F. and Mohamed M. N., Iraqi Journal of Statistical Sciences, 23:150-167, (2013). 